\title{
Microbiota for Healthy Ageing: The State of Art
}

\author{
Carla Pignatti ${ }^{1}$ and Stefania D'Adamo ${ }^{2,3 *}$ \\ ${ }^{1}$ Department of Biomedical and Neuromotor Sciences, University of Bologna, Italy \\ ${ }^{2}$ Department of Medical and Surgical Sciences, University of Bologna, Italy \\ ${ }^{3}$ Laboratory of Immunorheumatology and Tissue Regeneration, IRCCS Istituto Ortopedico Rizzoli, Italy \\ *Corresponding author: Stefania D’Adamo, Department of Medical and Surgical Sciences, Alma Mater Studiorum, University of \\ Bologna \& Laboratory of Immunorheumatology and Tissue Regeneration, IRCCS Istituto Ortopedico Rizzoli, Italy.
}

To Cite This Article: Carla Pignatti, Stefania D'Adamo, Microbiota for Healthy Ageing: The State of Art. 2020 - 11(1). AJBSR.MS.ID.001611. DOI: 10.34297/AJBSR.2020.11.001611.

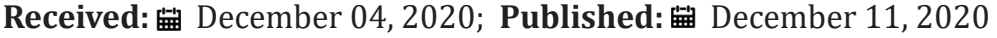

\begin{abstract}
Human microbiota is composed by trillions of bacteria which play a crucial role in host health maintenance and disease pathogenesis and whose composition is unique in each person. Environmental conditions and dietary habits can modulate the shape of microbiota that, in turn, may contribute to mediate the effect of dietary components on health status. The development of a stable and diverse gut microbiota is essential for various host physiologic functions such as immunoregulation, pathogen prevention, energy harvest, and metabolism.

Changes in microbial composition are often associated to the presence of common metabolic diseases such as obesity, type 2 diabetes, lipid disorders, metabolic syndrome. Gut microbiota composition is unique in each person and changes with age. While in adult's microbiota is generally characterized by high biodiversity, in the elderly it becomes compositionally unstable and less diverse, a condition known as dysbiosis. Dysbiosis is often linked to deterioration of the immune system, to a state of low-grade inflammation and might be causally related to sarcopenia.

Several gut bacterial species can ferment dietary fibers and produce short-chain fatty acids which exert beneficial effects on muscle mass during aging. Recently some phenolic compounds, as well as their microbic biotransformation metabolites have been shown to promote growth of specific microbial species known to be associated with an improved health status. Future studies are required to expand knowledges on implications of specific bioactive compounds and bacterial metabolites on age-related diseases. Moreover, an understanding of the role of micro-organisms colonizing other human body districts on host physiology should be taken in consideration.
\end{abstract}

Keywords: Microbiota, Ageing, Intestinal Dysbiosis, SCFA, Polyphenols, Biodiversity

\section{Mini review}

The human organism is a real ecosystem consisting of trillions of bacteria whose composition keeps changing in response to many factors such as dietary habits, seasonality, lifestyle, stress, antibiotics use, or diseases. Diet can modify the intestinal microbiota, which in turn has a profound influence on overall health [1]. This interaction can be beneficial or detrimental, depending on the relative identity and abundance of constituent bacterial populations. Nutrients and nutritional factors have an impact on evolutionarily conserved nutrient-sensing pathways that act on a network of interconnected downstream targets to regulate a range of molecular, cellular, physiological, and behavioral responses. In fact, environmental conditions and life-style can affect cellular pathways both directly and also indirectly by modulating the shape of microbiota that, in turn, may contribute to mediate the effect of dietary components on health status [2].

At all the ages, a healthy host-microorganism balance must be maintained in order to prevent disease onset. Unfortunately, the adoption of modern western dietary habits may lead to maladaptive changes of microbial strains which contribute to the development of metabolic diseases, such as obesity, diabetes, hypertension, dyslipidemia and finally of cancer [3]. There is vast preclinical basic and translational research suggesting multiple and complex mechanisms for a bacterial role in the obese state. Obesity is associated with deep gut microbial changes, which could alter 
the host's energy absorption and influence intestinal permeability, inflammation, immunity, and multiple other physiologic processes [4]. The "thrifty" gene hypothesis has postulated that evolutional pressure reinforced "thrifty" genes promoting the extraction of maximum calories (i.e., energy) from food sources in both humans and gut microcosm to increase survivability when food (i.e., energy) has been scarce [5].

Gut microbiota composition is unique in each person, even if malleable, and changes with age. In adults it is generally characterized by high biodiversity, while in the elderly the gut microbiota becomes compositionally unstable and less diverse, a condition known as dysbiosis, which may predispose to many age-related disabilities. Dysbiosis, is causally linked to a profound age-related remodeling and deterioration of the immune system, known as "immunosenescence" that is associated with a chronic low-grade inflammatory status, called "inflammaging" [6]. It has been suggested that the gut microbiota may influence sarcopenia, a syndrome which affects older individuals. Sarcopenia is described as depletion of muscle mass and reduction of muscle performance which both result from anabolic resistance or boosted protein catabolism [7].

Elderly microbiota is generally characterized by a decrease in species associated with short chain fatty acid (SCFA) production, especially butyrate and an increase in opportunistic pathogens [8]. The SCFA butyrate was shown to have beneficial effects on muscle mass in ageing mice, protecting them from muscle atrophy [9]. A gut-muscle axis between microbiota and sarcopenia has been suggested [10]. However very few studies have investigated the microbiome composition of sarcopenic individuals. Intake of lowcalories and nutrient-dense foods, well balanced in regulatory micronutrients, might favorably change microbiota composition and promote biodiversity, counteract age related intestinal dysbiosis and improve the nutritional status of elderly people [11]. The modulation of gut microbiota could help to improve a physiological and non-pathological aging process and, perhaps, to contrast the progression of degenerative mechanisms.

To better study the role of microbiota in aging process many researchers ventured in analyzing the gut microbiota of children or model organisms with the premature aging disorder called progeria. Mouse models of aging are affected by intestinal dysbiosis with an increase in the abundance of Proteobacteria and Cyanobacteria, and a decrease in the amount of Verrucomicrobia. After the transplantation of fecal microbiota from healthy mice, the life span was extended and the transplantation with the verrucomicrobia Akkermansia muciniphila was sufficient to exert beneficial effects [12]. These results suggested the existence of a real relationship between aging and the gut microbiota. Microbial metabolites, as SCFAs, affect gut-brain signaling and the immune response. The mechanisms by which the gut microcosm produces its effects on the brain are beginning to be understood [13]. Moreover, age-related microbiota changes have been implicated in the establishment of neurodegenerative diseases [6]. In particular, an increase in Bacteroides was linked to the levels of amyloid-beta $(A \beta)$ plaque deposition. This effect results normalized in calorie restriction (CR) conditions, suggesting a key role of gut microbiota composition in the development of age-related cognitive decline [14-16].

Key point in the research on anti-aging drugs has been the identification of bioactive molecules and phenolic compounds that could reproduce in adult humans the beneficial effects of CR, without the need to limit food intake. These compounds, named CR mimetics are able to act in an hermetic manner, stimulating several stress response pathways and induce pleiotropic effects that would produce health benefits and counteract many age-related diseases $[17,18]$. Some polyphenols have been shown to positively modulate the gut microbiota composition thus producing a prebiotic like effects [19]. As example, some plant-derived compounds may promote growth of specific gut microbial species including Akkermansia spp., Faecalibacterium spp. and Roseburia spp. that may provide beneficial health effects to the host $[19,20]$.

It is now generally accepted that health benefits from intake of polyphenols are linked, to a large extent, to their intestinal biotransformation and to production of bacterially derived metabolites. At present, most focus has been directed towards SCFAs and polyphenolic transformation products, owing to their various positive effects on immune and metabolic cues of relevance in life-style associated diseases [20]. Different phenolic compounds, especially flavonoids and their metabolites, have been shown to agonistically regulate peroxisome proliferator-activated receptor gamma (PPAR $\gamma$ ) activation to exert effects on inflammatory transcription factors, leading to suppression of inflammation and modification of phenotypes related to metabolic diseases [21]. However inconclusive effects on systemic pro-inflammatory markers (CRP and IL-6) as well as oxidative stress markers have been shown.

In Order to Better Understanding the Complex Microbiota-Host Relationships and Implication of This Interplay for Aged People, The Following Considerations Might Be Addressed

a) Many research in the field of polyphenol-microbiota interactions have been performed on cell cultures or in animal models with supraphysiological concentrations. Future studies are required to expand our knowledge on polyphenolic derived health effects in humans. In our opinion further consideration for future research should be also addressed toward the poor bioavailability of specific polyphenols and their biotransformation by certain gut bacteria. Individual 
differences in the gut microbiota, with particular regard to aged people might have a significant impact on the bioconversion of certain polyphenols. Future research should be designed to better understand the correlation between the gut microbiota, the ageing process and degenerative diseases typical of the elderly. This should be accompanied by a practical approach in the modulation of the gut microbiota easily applicable in the clinical context.

b) Still there is need of further studies in order to identify microbiota-related biomarkers of risk of obesity and metabolic diseases. Further basic and translational research and clinical trials are required to elucidate mechanisms, and specific probiotic and patients' types for the best achievable precision medicine approach to the obesity epidemic.

c) Investigation on the relevance of the gut microbiome to health and disease is in an early phase. The findings support the view that specific dietary regimens or food components, used alone or combined with the administration of combinations of probiotics that have been validated and approved by regulatory authorities (next generation, synbiotics) [22] may hold potential for enhancing public health.

d) Finally, we know that despite many steps have been made in the scientific research about the implication of gut microbiota in several diseases and in aging, many gaps remain to be filled on the contribute of micro-organisms colonizing other human body districts. It will be interesting to understand whether vaginal, oral, skin, nasal microbiota could contribute to host physiology. and, when altered, may be able to influence pathologies affecting distant organs and tissues, fueling inflammaging and contributing to immunosenescence, to our knowledge only a recent review takes in consideration this inexplorated field [23].

\section{Conflict of Interest}

The authors declare no conflict of interest.

\section{References}

1. Requena T, Martinez-Cuesta MC, Pelaez C (2018) Diet and microbiota linked in health and disease. Food Funct 9(2): 688-704.

2. Pignatti C, D’Adamo S, Stefanelli C, Flamigni F, Cetrullo S, et al. (2020) Nutrients and Pathways that Regulate Health Span and Life Span. Geriatrics (Basel) 5(4): 95.

3. Lynch SV, Pedersen O (2016) The Human Intestinal Microbiome in Health and Disease. N Engl J Med 375(24): 2369-2379.

4. Rastelli M, Knauf C, Cani PD (2018) Gut Microbes and Health: A Focus on the Mechanisms Linking Microbes, Obesity, and Related Disorders. Obesity (Silver Spring) 26(5): 792-800.

5. Higginson AD, Mc Namara JM, Houston AI (2016) Fatness and fitness: exposing the logic of evolutionary explanations for obesity. Proceedings of Biological Sciences 283(1822).
6. Mangiola F, Nicoletti A, Gasbarrini A, Ponziani FR (2018) Gut microbiota and aging. European Review for Medical and Pharmacological Sciences 22: 7404-7413.

7. Cruz-Jentoft AJ, Baeyens JP, Bauer JM, Boirie Y, Cederholm T, et al. (2010) Sarcopenia: European consensus on definition and diagnosis: Report of the European Working Group on Sarcopenia in Older People. Age and Ageing 39(4): 412-23.

8. Biagi E, Candela M, Turroni S, Garagnani P, Franceschi C, et al. (2013) Ageing and gut microbes: Perspectives for health maintenance and longevity. Pharmacol Res 69(1): 11-20.

9. Walsh ME, Bhattacharya A, Sataranatarajan K, Qaisar R, Sloane L, et al. (2015) The histone deacetylase inhibitor butyrate improves metabolism and reduces muscle atrophy during aging. Aging Cell 14(6): 957-970.

10. Ticinesi A, Lauretani F, Milani C, Nouvenne A, Tana C, et al. (2015) Aging Gut Microbiota at the Cross-Road between Nutrition, Physical Frailty, and Sarcopenia: Is There a Gut-Muscle Axis? Nutrients 9(12): 1303.

11. Kim S, Jazwinski SM (2018) The Gut Microbiota and Healthy Aging: A Mini-Review. Gerontology 64(6): 513-520.

12. Barcena C, Valdes-Mas R, Mayoral P, Garabaya C, Durand S, et al. (2019) Healthspan and lifespan extension by fecal microbiota transplantation into progeroid mice. Nat Med 25(8): 1234-1242.

13. Mohajeri MH, La Fata G, Steinert RE, Weber P (2018) Relationship between the gut microbiome and brain function. Nutr Rev 76(7): 481496.

14. Harach T, Marungruang N, Duthilleul N, Cheatham V, Mc Coy KD, et al. (2017) Reduction of Abeta amyloid pathology in APPPS transgenic mice in the absence of gut microbiota. Sci Rep 7: 41802.

15. Minter MR, Zhang C, Leone V, Ringus DL, Zhang X, et al. (2016) Antibiotic-induced perturbations in gut microbial diversity influences neuro-inflammation and amyloidosis in a murine model of Alzheimer's disease. Sci Rep 7(1): 10411.

16. Cox LM, Schafer MJ, Sohn J, Vincentini J, Weiner HL, et al. (2019) Calorie restriction slows age-related microbiota changes in an Alzheimer's disease model in female mice. Sci Rep 9(1): 17904.

17. Ingram DK, Roth GS (2015) Calorie restriction mimetics: Can you have your cake and eat it, too? Ageing Res Rev 20: 46-62.

18. Madeo F, Carmona-Gutierrez D, Hofer SJ, Kroemer G (2019) Caloric Restriction Mimetics against AgeAssociated Disease: Targets, Mechanisms, and Therapeutic Potential. Cell Metab 29(3): 592-610.

19. Tomas-Barberan FA, Selma MV, Espin JC (2016) Interactions of gut microbiota with dietary polyphenols and consequences to human health. Curr Opin Clin Nutr Metab Care 19(6): 471-476.

20. Danneskiold-Samsøe NB, Dias de Freitas Queiroz Barros H, Santos R, Bicas JL, Cazarin CBB, et al. (2019) Interplay between food and gut microbiota in health and disease. Food Research International 115: 2331.

21. Wang L, Waltenberger B, Pferschy-Wenzig EM, Blunder M, Liu X, et al. (2014) Natural product agonists of peroxisome proliferator-activated receptor gamma (PPARgamma): a review. Biochem Pharmacol 92(1): 73-89.

22. Cani PD, de Vos WM (2017) Next-Generation Beneficial Microbes: The Case of Akkermansia muciniphila. Front Microbiol 8:1765.

23. Santoro A, Zhao J, Wu L, Carru C, Biagi E, et al. (2020) Microbiomes other than the gut: inflammaging and age-related diseases. Semin Immunopathol 42(5): 589-605. 